\title{
Mesenchymal stem cell therapy of hepatocellular carcinoma in rats: Detection of cell homing and tumor mass by magnetic resonance imaging using iron oxide nanoparticles
}

\author{
Mamdooh Faidah 1, B-D, F, Abdulwahab Noorwali ${ }^{2,3, A-C, F}$, Hazem Atta ${ }^{4,5, A-F}$, Naushad Ahmed ${ }^{6, B-F}$, Hamid Habib, A, D-F, Laila Damiati, B-F, \\ Najlaa Filimban ${ }^{3, B-D}$, Mihal Al-qriqri, B-D, Soheir Mahfouz ${ }^{8, B-F}$, Mohamad Nidal Khabaz ${ }^{9}$, B-F \\ ${ }^{1}$ Department of Medical Laboratory, College of Health Sciences, King Abdulaziz University, Jeddah, Saudi Arabia \\ ${ }_{2}^{2}$ Department of Clinical Biochemistry, College of Medicine, King Abdulaziz University, Jeddah, Saudi Arabia \\ ${ }^{3}$ Stem Cell Unit, King Fahd Center for Medical Research, King Abdulaziz University, Jeddah, Saudi Arabia \\ ${ }^{4}$ Department of Clinical Biochemistry, Faculty of Medicine, Rabigh, King Abdulaziz University, Jeddah, Saudi Arabia \\ ${ }^{5}$ Department of Medical Biochemistry, Faculty of Medicine, Cairo University, Egypt \\ ${ }^{6}$ Department of Radiology, King Abdul Aziz University Hospital, King Abdulaziz University, Jeddah, Saudi Arabia \\ ${ }^{7}$ Department of Pediatrics, Faculty of Medicine, King Abdulaziz University, Jeddah, Saudi Arabia \\ ${ }^{8}$ Department of Pathology, Faculty of Medicine, Cairo University, Egypt \\ ${ }^{9}$ Department of Pathology, Rabigh Faculty of Medicine, King Abdulaziz University, Jeddah, Saudi Arabia \\ A - research concept and design; $B$ - collection and/or assembly of data; $C$ - data analysis and interpretation; \\ $\mathrm{D}$ - writing the article; $\mathrm{E}$ - critical revision of the article; $\mathrm{F}$ - final approval of the article
}

\section{Address for correspondence}

Hazem Atta

E-mail:hmaatta@kau.edu.sa

\section{Funding sources}

This work was supported by grant number (EM/33/2) from the Deanship of Scientific Research, King Abdulaziz University, Jeddah, Saudi Arabia, awarded to Abdulwahab Noorwali and Hazem Atta.

\section{Conflict of interest}

None declared

\section{Acknowledgements}

This work is a part of the of the research activities of the Scientific Research Group for Tissue Engineering and Regenerative Medicine, Faculty of Medicine, King Abdulaziz University, Jeddah, Saudi Arabia. The authors acknowledge the continuous support and valuable guidance of the Deanship of Scientific Research (DSR) of King Abdulaziz University, Jeddah, Saudi Arabia.

Received on November 12, 2015

Reviewed on February 18, 2016

Accepted on December 6, 2016

DOI

$10.17219 /$ acem/67563

Copyright

Copyright by Author(s)

This is an article distributed under the terms of the Creative Commons Attribution Non-Commercial License (http://creativecommons.org/licenses/by-nc-nd/4.0/)

\begin{abstract}
Background. Bone marrow-derived mesenchymal stem cells (MSCS) are reported to improve hepatic fibrosis, and may impact the signaling mechanisms leading to the induction of hepatocellular carcinoma (HCC) in animal models of liver cirrhosis.

Objectives. The aim of this study was to clarify and explain the therapeutic role played by MSCs in hepatic cirrhosis and HCC by tracking them using nanoparticles.

Material and methods. Liver cirrhosis and HCC were established in rats with the use of carbon tetrachloride and diethylnitrosamine injection. Magnetic resonance imaging (MRI) was used to track nanoparticlelabeled MSCs in the intact animal following injection and to monitor the changes in the hepatic parenchyma.

Results. Labeling of MSCS with iron oxide nanoparticles did not adversely affect their viability and proliferation. MRI indicated a significant reduction in tumor mass in the labeled MSCs group compared to the control group. Histopathologic examination of the liver, following MSCs treatment, showed an apparently normal looking liver with no evidence of neoplastic cellular changes. The biochemical results support these findings.

Conclusions. This work documents that MSCS could be labeled with nanoparticles and traced in normal and cirrhotic liver and in liver with HCC in animals using MRI. MRI monitors the homing and localization of MSCs in the liver. MSCs infusion in animal models of cirrhosis and carcinoma may prove to be useful in limiting the cirrhotic process. Also, it may have a possible therapeutic potential on the carcinogenic process.
\end{abstract}

Key words: liver cirrhosis, nanoparticles, stem cells, hepatocellular carcinoma 


\section{Introduction}

Hepatocellular carcinoma (HCC) is a widespread, treatment-resistant malignancy, culminating in various molecular etiologies. ${ }^{1}$ It is a significant worldwide health problem with more than half a million cases diagnosed annually. ${ }^{2}$ Current data indicate that during transformation to cancer, 2 main pathogenic mechanisms occur: cirrhosis after tissue damage and mutations in oncogenes or tumor suppressor genes. Both mechanisms are followed by changes in important intracellular signals. Targeting these signaling pathways may have a therapeutic perspective, as it may help to reverse, delay or prevent carcinogenesis. ${ }^{1}$

Mesenchymal stem cells (MSCs) are being investigated as possible anti-cancer agents indicating a potential to be used to treat different types of malignant tumors. In a review by Shah, it was reported that stem cell therapy holds a remarkable promise for the treatment of human disease. ${ }^{3}$ MSCs possess inherent migratory properties toward tumor cells. This may enable their use as a means for delivering effective, targeted therapy to tumor cells. They could be programmed to express anti-proliferative, pro-apoptotic, anti-angiogenic agents, targeting different cancer types.

Qiao and coworkers showed that human mesenchymal stem cells (hMSCs) can localize tumor sites, preventing the proliferation of tumor cells. ${ }^{4}$ Others reported that hMSCs, in high proportions, inhibited the proliferation of HepG2 human liver cancer cell lines through mitogen-activated protein kinase (MAPK) and phosphatidylinositol 3-kinase (PI3K) signaling pathways. ${ }^{5}$ The stem cells seem to create signals leading to the inhibition of cell proliferation and stimulation of differentiation. ${ }^{6}$ Tumor cells are capable of secreting factors that can attract hMSCs to the tumor site. ${ }^{7}$ MSCs were proven to regenerate damaged livers.

Currently, super paramagnetic iron oxide nanoparticles (SPION) are safely used for cell tracking. ${ }^{8}$ In vivo tracking and visualization of the transplanted stem cells is an essential means for monitoring their tissue localization and may confirm their therapeutic potential. ${ }^{9}$

This study aimed to confirm the role played by MSCs in the amelioration of hepatic pathology in the rat model of cirrhosis/HCC, through MRI tracking of cells labeled with iron oxide nanoparticles. Also, to elucidate the molecular signals responsible for the transformation of liver cells to cancer. The combination of cell labeling and MRI tracking in a time dependent manner with close follow-up of the lesion size and histologic changes provide a novel, more objective assessment of the role of MSCs in disease control.

\section{Material and methods}

The approval of the biomedical research ethics committee of the Faculty of Medicine, King Abdulaziz
University (KAU), Jeddah, was obtained prior to the study. All the institution guidelines for conducting animal research were followed throughout this work.

\section{Preparation of bone marrow derived MSCs}

The method used was a modification of that of Abdel Aziz et al. ${ }^{10}$ Bone marrow was collected from the long bones of male rats, and washed with Dulbecco's Modified Eagle's medium (DMEM, GIBCO/BRL) supplemented with $10 \%$ fetal bovine serum (GIBCO/BRL) and 1\% penicillin-streptomycin (GIBCO/BRL). Unattached cells were removed daily, and adherent cells were kept in the incubator at $37^{\circ} \mathrm{C}$ in $5 \%$ humidified $\mathrm{CO}_{2}$ for $10-15$ days as primary culture. When the cells reached $80-90 \%$ confluence, they were washed twice with phosphate buffer saline (PBS), and trypsinized with $0.25 \%$ trypsin in $1 \mathrm{mM}$ EDTA (GIBCO/BRL) for $5 \mathrm{~min}$ at $37^{\circ} \mathrm{C}$. Cells were then centrifuged and resuspended in $50 \mathrm{~cm}^{2}$ culture flasks (falcon), and incubated (first passage cultures). MSCs in culture were characterized by their adhesiveness and spindle shape and by flow cytometry.

\section{Flow cytometry analysis}

MSCs were characterized using cell surface markers to confirm their identity by being CD29+ and CD45using specific monoclonal antibodies (Alexa Fluor 488 anti-mouse/rat CD29 antibody, cat\# 102212 and PE Antirat CD45 antibody, cat\# 202207, BioLegend, California, USA). $5 \times 10^{5}$ cells were mixed with $10 \mu \mathrm{L}$ of the fluorescent-labeled monoclonal antibodies, then incubated in the dark at $2-8^{\circ} \mathrm{C}$ for $30 \mathrm{~min}$. They were washed twice with phosphate buffered saline (PBS), and the pellet was re-suspended in PBS and analyzed immediately on FACS Aria III (Becton Dickinson, New Jersey, USA).

\section{Labeling of MSCs with iron oxide nanoparticles}

Fluid MAG-nanoparticles (Chemicell, Berlin, Germany) are ferrofluid, 200-nm-diameter particles composed of a magnetite core coated by hydrophilic polymers protective against aggregation. ${ }^{11}$ To improve the ability of these particles to be endocytosed by MSCs, poly-l-lysine was utilized as a prospective vehicle for iron oxide nanoparticle transport into cells. ${ }^{12}$

MSCs were counted, then seeded in micro-well plates at the density of $10^{5}$ cells/well in a culture medium. They were then incubated with medium containing $25 \mu \mathrm{g}$ $\mathrm{Fe} / \mathrm{mL}$ and $0.75 \mathrm{mg} / \mathrm{mL}$ Poly-l-lysine for $24 \mathrm{~h}$.

To confirm MSCs labeling with iron oxide nanoparticles by transmission electron microscopy, cells were trypsinized, washed 3 times with PBS, centrifuged, and fixed in reagent (glutaraldehyde). The viability of unlabelled MSCs and those incubated with SPION was analyzed 
by Micro culture Tetrazolium Assay (MTT, Sigma Chemical Company, St Louis, USA).

\section{Animal model for liver cirrhosis and hepatocellular carcinoma}

Female albino rats, bred in the animal facilities of King Fahd Center for Medical Research (King Abdulaziz University, Jeddah) were included in the study. They ranged in age between 16 and 24 weeks. Their body weight was $120-150 \mathrm{~g}$.

HCC was induced by a single intra-peritoneal injection of Diethylnitrosamine (DENA, Sigma, USA), containing $200 \mathrm{mg} / \mathrm{kg}$ body weight. ${ }^{13}$

Liver cirrhosis was induced by subcutaneous injection of $3 \mathrm{~mL} / \mathrm{kg}$ body weight of carbon tetrachloride $\left(\mathrm{CCl}_{4}\right)$ in castor oil. ${ }^{13}$ Injection was carried out 3 times in the $1^{\text {st }}$ week, then twice per week for 12 weeks. Animals were divided equally into 4 groups (10 animals each). The $1^{\text {st }}$ group is a control group of 10 normal rats that received castor oil subcutaneously. Five of them received labeled MSCs after 12 weeks to serve as a control for the radiology study. Cells were injected into the rattail vein $\left(3 \times 10^{6}\right.$ cells in PBS). Cells were injected twice with 1-week interval between injections. The same cell count and injection protocol was followed in all groups. The $2^{\text {nd }}$ group (no treatment group) included rats, which received MSCs vehicle (PBS) only after induction of HCC by DENA and $\mathrm{CCl}_{4}$. The $3^{\text {rd }}$ group included rats, which received MSCs after induction of $\mathrm{HCC}$ by DENA and $\mathrm{CCl}_{4}$ (treatment group). The $4^{\text {th }}$ group included rats, which received iron oxide nanoparticles-labeled MSCs after induction of $\mathrm{HCC}$ by DENA and $\mathrm{CCl}_{4}$. Rats from this group were included in the radiological study only to follow the disease progression by MRI.

\section{Histological examination}

Liver tissue sections were fixed in buffered formalin, paraffin-embedded, then cut at 5- $\mu \mathrm{m}$ sections and stained with hematoxylin and eosin. Liver tissue was analyzed for the $\mathrm{HCC}$ induction and progression of treatment with MSCs.

\section{Magnetic resonance imaging}

Localization and tracking of the migration of stem cells to the site of the lesions was observed using iron nanoparticles. MRI was used to track the injected cells in a time dependent manner.

The scans were performed on a Biospec 94/30 USR research MR scanner utilizing a T2 Turbo RARE (rapid acquisition with relaxation enhancement). ${ }^{14,15} \mathrm{~T} 2$ weighted MR sequences are best suited to evaluate the liver in cases of cirrhosis and hepatocellular carcinoma. ${ }^{16}$ The base component of nanoparticles is iron oxide, which being para-magnetic, causes shortening of the T2 signal. ${ }^{17,18}$
Two separate time course MR imaging were employed. Axial T2 Turbo RARE images were performed on rats with nanoparticle labeled MSCs (Fig. 5) on days 5, 7, 9 and 13 after the $1^{\text {st }}$ dose; and days 2 and 6 after the $2^{\text {nd }}$ dose of the nanoparticle labeled stem cells. Additionally, in the rats with nanoparticles, Axial T2 RARE scans were performed at time intervals of $30 \mathrm{~min}$ after injection of the nano-labeled stem cells up to $90 \mathrm{~min}$. Care was taken to ensure that the sequence parameters were kept standard in all scans (TR - $110 \mathrm{~ms}$, TE - $27 \mathrm{~ms}$, FA - 180 degrees, NEX 4).

\section{Biochemical investigations}

Serum activities of enzymes aspartate aminotransferase (AST) and alanine aminotransferase (ALT) were measured by the Flex ${ }^{\circledR}$ reagent cartridges using the Dimension Vista ${ }^{\circledR}$ system analyzer (Siemens Healthcare Diagnostics Incorporated, Newark, USA). Serum level of rat $\alpha$-fetoprotein was measured by enzyme linked immunosorbent assay (ELISA) using a kit purchased from USCN Life Science (Wuhan, China).

\section{Molecular studies}

\section{Ribonucleic acid (RNA) isolation}

Total RNA was isolated from liver tissue with the MagNA Pure Compact (Roche) System according to the manufacture's protocol for RNA purification (Cat\# 04802993001).

\section{Reverse transcription polymerase chain reaction (RT-PCR) and real time PCR assay}

In brief, complementary deoxyribonucleic acid (cDNA) was generated from total RNA using High-Capacity cDNA Reverse Transcription (Applied Biosystem, and Cat \# 4375222). RT-PCR was performed in duplicate on AB StepOne Plus System using TaqMan ${ }^{\circledR}$ Universal PCR Master Mix. The total reaction volume was $20 \mu \mathrm{L}$. Expression patterns were evaluated for cyclin D1, beta-catenin and survivin, using TaqMan gene expression assays (Applied Biosystems; Rn00432360, Rn00584431, and Rn00574012, respectively). Real Time PCR was then performed. Cycling condition included: initiation step at $95^{\circ} \mathrm{C}$ for $2 \mathrm{~min}$, denaturation at $95^{\circ} \mathrm{C}$ for $1 \mathrm{~min}$, annealing at $60^{\circ} \mathrm{C}$ for $30 \mathrm{~s}$, extension at $72^{\circ} \mathrm{C}$ for $30 \mathrm{~s}$, followed by 40 cycles. Relative gene expression data was analyzed using a mathematical equation. Hypoxanthine phosphoribosyltransferase 1 (Hprt1, AB, Rn01527840) was used as endogenous control for normalization.

\section{Statistical analysis}

Statistical analysis for all studied parameters was carried out using the Statistical Package for Social Sciences v. 16 (SPSS, Inc. Chicago, USA). Significance level was 
Fig. 1. Flow cytometric analysis of rat MSCs
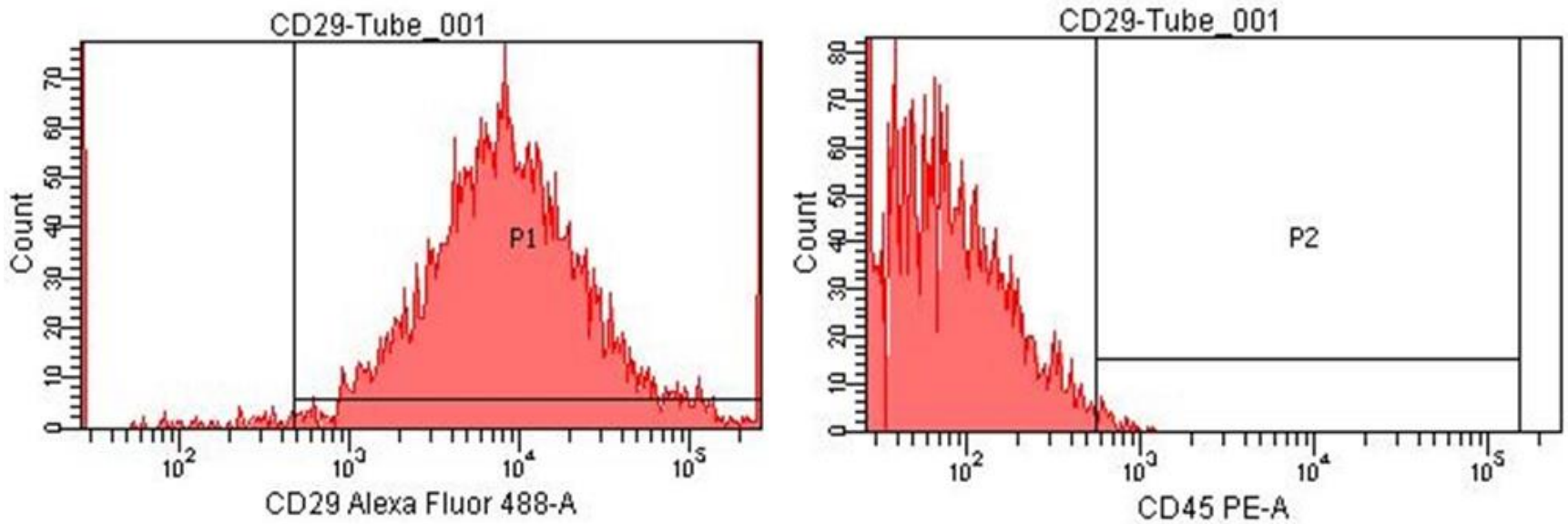

The cells were CD29+ (A) and CD 45- (B). MSCs were stained using Alexa Fluor 488 anti-mouse/rat CD29 antibody (A. Positive) and PE Anti-rat CD45 antibody (B. Negative). These molecules are surface markers for the characterization of MSCs.

Fig. 2. Detection of nanoparticles inside labeled MSCs using transmission electron microscope

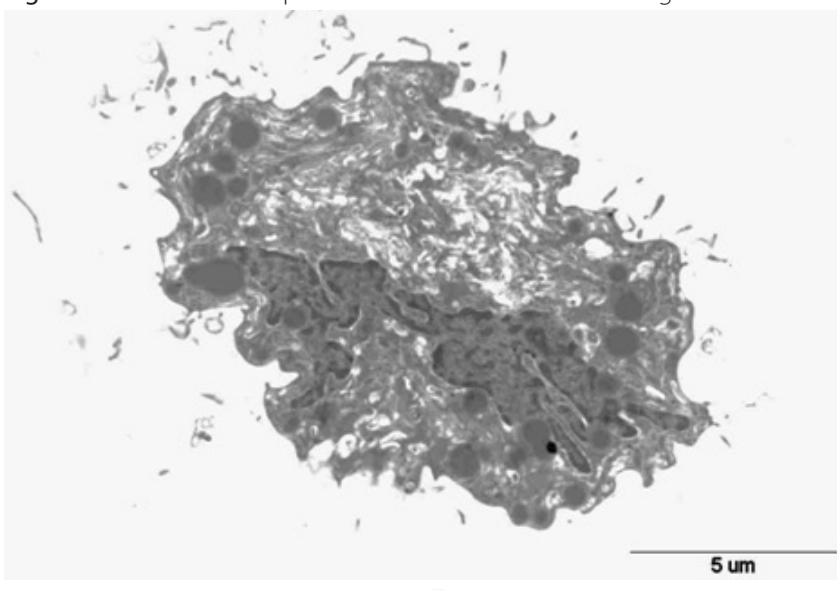

A

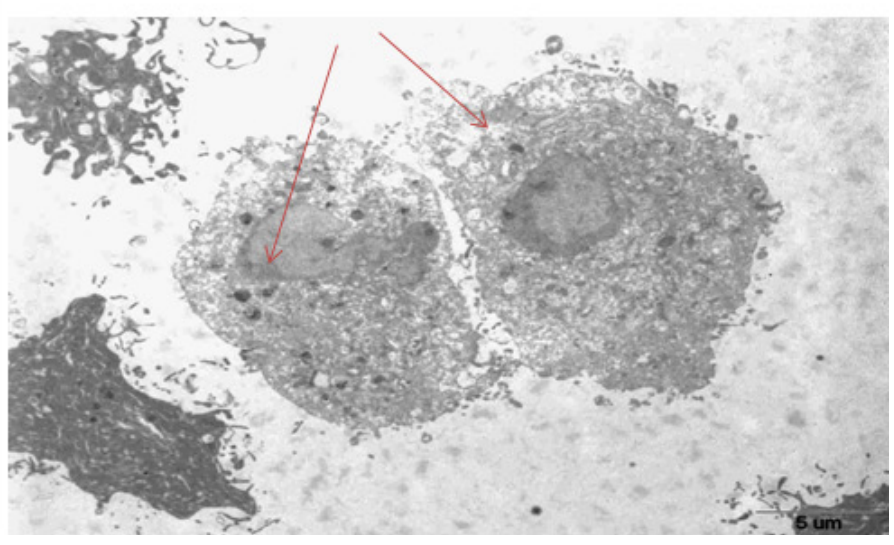

B

A - normal mesenchymal stem cell that did not receive nanoparticles ( $\times 4600)$; B - iron oxide nanoparticles detected inside the cytoplasm of mesenchymal stem cells (red arrows, $\times 2600$ ).

set at $<0.05$. Univariate analysis of data was carried out by applying measures of central tendency to describe quantitative variables, mean and standard deviation (SD). A one-way analysis of variance (ANOVA) was used to test for difference in the readings between the control, no treatment and treatment groups. When significance was found, it was followed by a post hoc test to determine significant inter-group difference.

\section{Results}

Isolated rat bone marrow MSCs were identified by their fusiform shape. Flow cytometry confirmed their identity by being $\mathrm{CD}_{29^{+}}$and $\mathrm{CD} 45^{-}$(Fig. 1).

Labeling of MSCs with iron oxide nanoparticles was successful as detected by transmission electron microscope (Fig. 2). Viability of MSCs was not adversely affected by iron as proven by viability assay (Fig. 3 ).

\section{Histology findings}

The investigators succeeded in developing the desired pathology of cirrhosis (Fig. 4. C, D) and hepatocellular carcinoma (Fig. 4. E, F) in the rat model. This is in comparison to the normal liver shown in Fig. 4 (A, B). The liver restored its original histologic architecture after treatment with MSCs (Fig. 4. G, H), which shows apparently normal looking liver trabeculae and sinusoids with no evidence of preneoplastic or neoplastic cellular changes.

\section{Magnetic resonance imaging findings}

A sequential fall in the signal intensity of the liver was detected in the scan performed at 30 min intervals (Fig. 5). Given the paramagnetic properties of nanoparticles, there is a fall in signal associated with SPION-labeled MSCs reaching the liver. This fall in signal intensity was noted for a further 48 hours after which the signals increased 
Table 1. Comparison of mean levels of serum activity of alanine aminotransferase (ALT), aspartate aminotransferase (AST) and serum level of a-fetoprotein in the studied groups

\begin{tabular}{|c|c|c|c|}
\hline Study groups & $\begin{array}{c}\text { ALT U/L } \\
\text { mean } \pm S D\end{array}$ & $\begin{array}{l}\text { AST U/L } \\
\text { mean } \pm S D\end{array}$ & $\begin{array}{c}\text { a-fetoprotein } \mathrm{ng} / \mathrm{mL} \\
\text { mean } \pm \mathrm{SD}\end{array}$ \\
\hline Control group & $82.00 \pm 3.91$ & $41.25 \pm 3.50$ & $0.278 \pm 0.07$ \\
\hline No treatment group & $304.25 \pm 77.69^{a}$ & $545.50 \pm 81.32^{\mathrm{a}}$ & $27.435 \pm 2.97^{a}$ \\
\hline Treatment group & $89.25 \pm 27.62^{b}$ & $182.00 \pm 68.10^{b, c}$ & $1.261 \pm 0.88^{b, c}$ \\
\hline p-value (ANOVA) & $0.000^{*}$ & $0.000^{*}$ & $0.000^{*}$ \\
\hline
\end{tabular}

$\mathrm{p}$-value significant at $<0.05$; $\mathrm{p}$-value significant between all tested groups; a $\mathrm{p}$-value significant between control and the untreated group; $\mathrm{b}$ - $\mathrm{p}$-value significant between untreated group and the treatment group; c - p-value significant between control and treatment group.

Fig. 3. Effect of iron oxide nanoparticles on the viability of rat MSCs

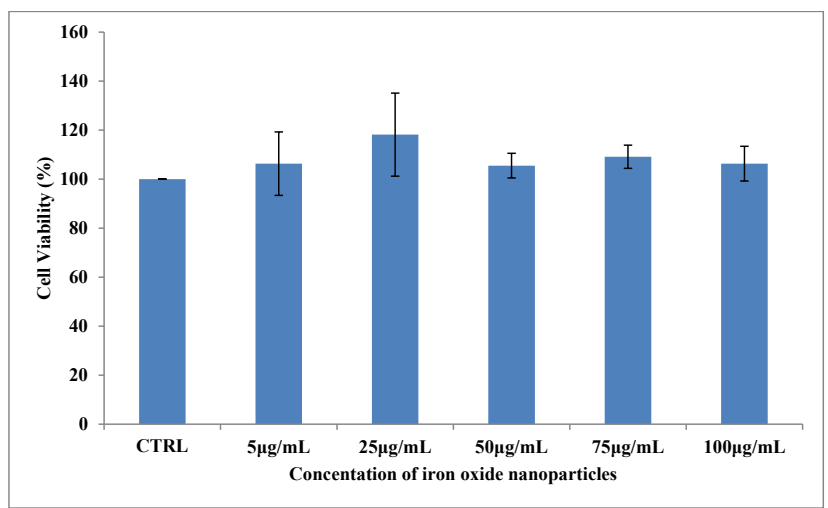

(Fig. 6). This is most likely due to a combination of the nanoparticles excretion and the regeneration of liver parenchyma due the effect of the infused MSCs. A representative nodule was chosen in each of the scans, and its dimensions were measured over time using a vendor provided calipers tool with measurements in millimeters. The lesion showed a significant reduction in dimensions (Fig. 7).

\section{Biochemical findings}

Rats treated with MSCs showed a significant decrease in serum level of $\alpha$-fetoprotein, and serum activities of ALT and AST after MSCs therapy, when compared to the diseased untreated group (Table 1). The same effect was observed with both labeled and unlabeled cells. However, AST activity and the level of $\alpha$-fetoprotein remained significantly higher in the MSCs treated group when compared to the control group.

\section{Molecular findings}

Gene expression of survivin, cyclin D1 and beta-catenin were compared before and after MSCs therapy (Fig. 8). A decrease in the gene expression of cyclin D1 was observed with stem cell therapy. This decrease was not statistically significant when compared to the control and the no treatment groups. Gene expression of beta-catenin was significantly lower in the treatment group compared to the control and the no treatment groups.
Fig. 4. Normal liver tissue (A, B), cirrhotic liver (C, D) and hepatocellular carcinoma $(E, F)$

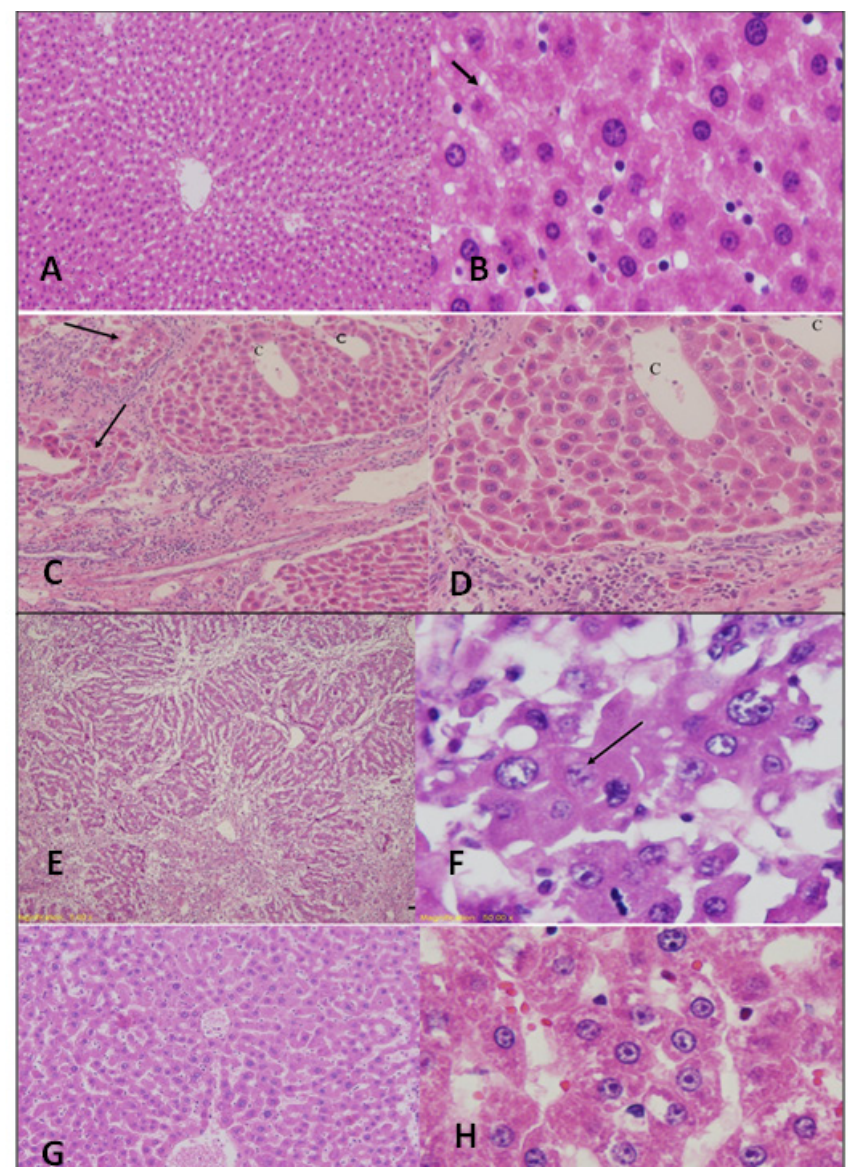

Pictures $A$ and $B$ show normal liver tissue in control group $(\mathrm{HE} \times 100$ and $\times 400$ ). Pictures $C$ and $D$ reveal cirrhotic livers; there is an abnormal arrangement of hepatocytes around 2 central veins (arrow), and the nodule is totally surrounded by inflamed fibrous tissue septa with thickening of liver plates and distortion of portal tracts ( $H E \times 200$ and $\times 400$ ). Pictures $E$ and $F$ are presenting hepatocellular carcinoma; there are areas of invasive cells assuming a trabecular pattern (E), whereas in picture D there are pleomorphic hepatocytes with hyperchromatic nuclei, prominent large nucleoli, scattered mitotic figures (arrow) and high N/C ratios $(\mathrm{HE} \times 100$ and $\times 400$ ). Following treatment with MSCs ( $\mathrm{G}$ and $H)$, the liver shows apparently normal looking liver trabeculae and sinusoids with no evidence of preneoplastic or neoplastic cellular changes $(\mathrm{HE} \times 200-\times 400)$ 
Fig. 5. Sequential fall in the signal intensities at scan intervals of $30 \mathrm{~min}$, denoting the paramagnetic effect of the nanoparticles

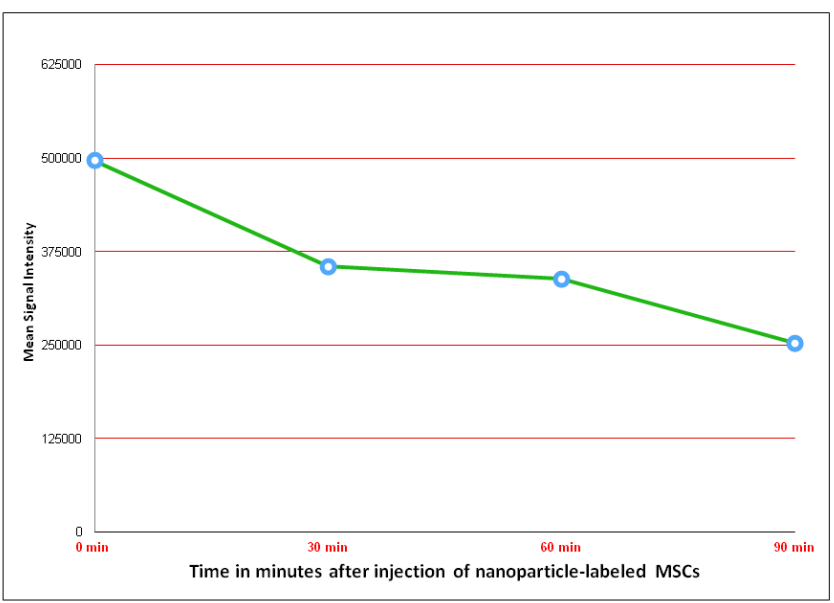

Fig. 6. Changes in signal intensity of the liver in the labeled rat (decrease for up to $24 \mathrm{~h}$ after which an increase in signal intensity was detected)

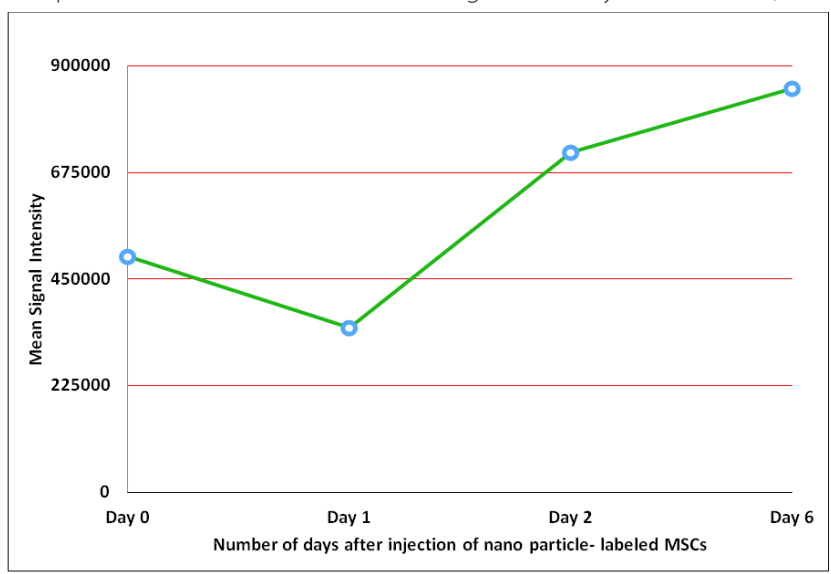

\section{Discussion}

This work aimed at investigating the therapeutic potential of MSCs on HCC in the rat model of liver cirrhosis/HCC. The work intended to document and correlate radiologic evidence with both histological and molecular evidence of the reported healing effect of MSCs.

MSCs were isolated from the bone marrow of male rats and propagated in culture. MSCs were identified by their morphology and flow cytometry. These results are in agreement with those of Abdel Aziz et al., who developed a rat model of cirrhosis and HCC. ${ }^{13}$ Scientists isolated MSCs from the bone marrow of rats and were able to use them to treat both liver cirrhosis and HCC.

The MSCs were labeled with iron oxide nanoparticles, which did not affect their viability or proliferative ability. These results are in agreement with those of Wang et al., 2009. ${ }^{14}$ They found that growing Adipose tissue derived MSCs (ASCs) in SPION-containing medium did not adversely affect the survival rate (97-99\%). The labeled cells continued to function normally and to express cell factors, and were able to trans-differentiate. The study concluded
Fig. 7. Axial T2 Turbo RARE images of rat livers infused with nanoparticlelabeled MSCs

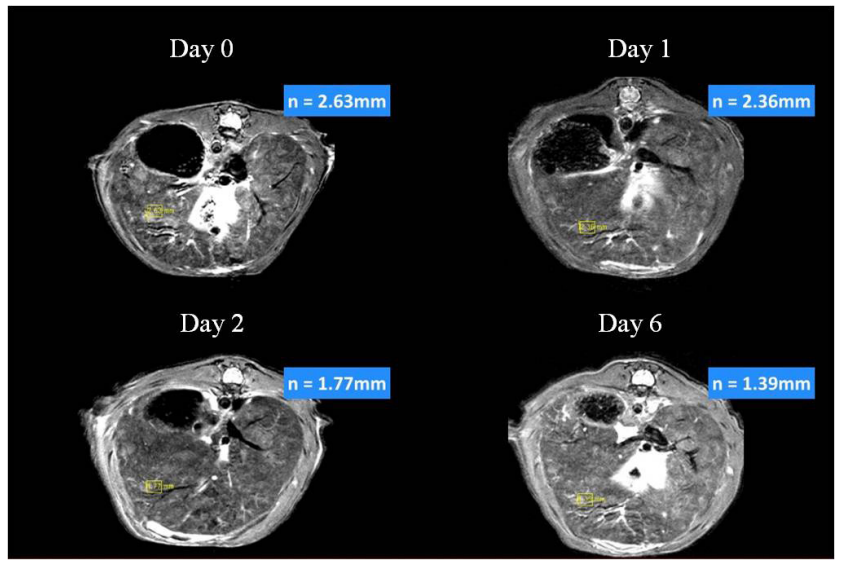

A representative nodule was chosen and measured in equivalent cuts in serial scans performed on days 0, 1, 2 and 6 after MSCs injection. The serial cuts demonstrated interval decrease in dimensions (Day $0=2.63 \mathrm{~mm}$, Day $1=2.36 \mathrm{~mm}$, Day $2=1.77 \mathrm{~mm}$, Day $6=1.39 \mathrm{~mm}$ ).

Fig. 8. Gene expression of survivin, cyclin D1 and beta-catenin with and without MSCs treatment

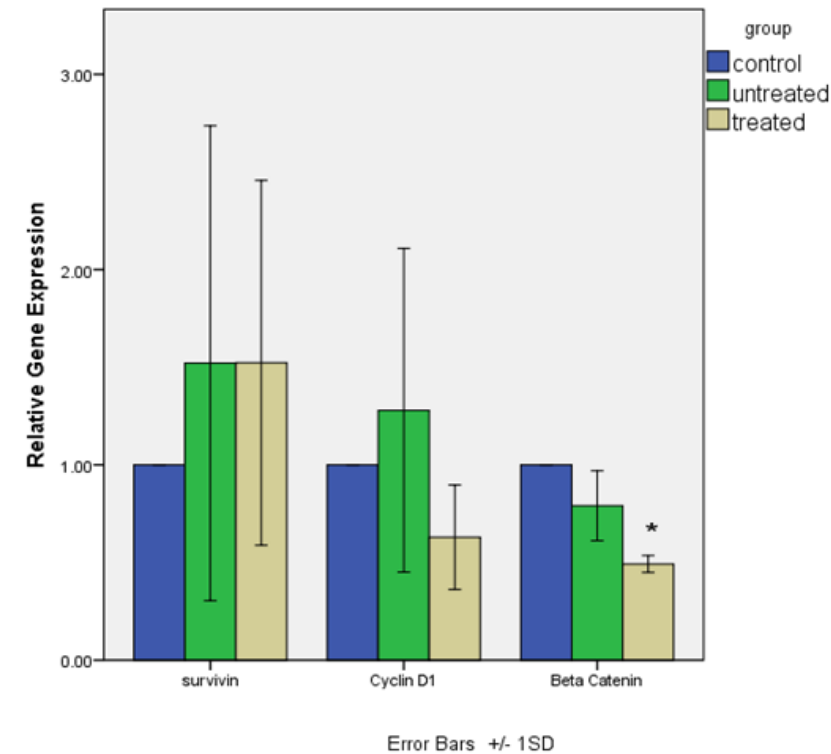

The expression of these genes was assessed by real-time polymerase chain reaction. MSCs therapy decreased the expression of cyclin D1 and beta-catenin. Significant decline was observed with beta-catenin.

that SPION did not affect viability, trans-differentiation potential or cell-factor secretion of ASCs. The results also agree with those of Zhao et al., who transplanted SPION labeled MSCs via the rat tail vein and the portal vein into fibrotic rat livers, then visualized them by MRI. ${ }^{19}$

In this work, MSCs injection improved hepatic pathology as depicted by the diminishing cirrhosis and the signs of recovery in the affected liver. These findings agree with those of Abdel Aziz et al. and Zhao et al. ${ }^{13,19}$ The biochemical, molecular and radiologic findings confirmed the recovery of the injured liver. The mean serum level of $\alpha$-fetoprotein showed a significant decline in the animals that received MSCs therapy compared 
to the no treatment group. Serum activities of ALT and AST significantly declined following MSCs therapy. These results are in agreement with those of Zhao et al. ${ }^{19}$

Time course MRI was adopted to confirm the homing of MSCs to the injured liver, and to document their therapeutic effects by correlating the radiologic findings to the hepatic pathology. Figure 5 follows the labeled MSCs into the injured liver by MRI. There was an initial decline in signal intensity, coinciding with MSCs injection and continuing for 24-48 h. This was followed by a gradual increase in signal intensity till the animals were sacrificed. The decline in signal intensity could be explained by the homing of iron-labeled cells into the liver in the first 24-48 h following IV injection. The increase in intensity thereafter may be explained by the improvement in liver pathology associated with MSCs injection, as well as the clearance of iron from the injected cells, possibly through extrusion and biliary excretion or engulfment by hepatic Kupffer cells. Figure 7 is focused on measuring the size of a representative focal lesion in the liver and the progressive decline in its diameter associated with stem cell therapy. This confirms and correlates with the amelioration of hepatic pathology.

Bos et al. used magnetic resonance imaging (MRI) for tracking SPION-labeled MSCs. ${ }^{20}$ These labeled cells were transfused into the renal arteries and portal veins of rats. SPION labeling had no hazardous effect on the viability and differentiation of the injected cells. The liver showed diffuse granular appearance associated with stem cell infusion. The study concluded that MRI is important for monitoring of SPION-labeled MSCs in vivo, in the liver. The results also agree with those of Zhao et al., who used MRI to confirm that SPION-labeled MSCs colonized the liver. ${ }^{19}$ They concluded that in vivo MRI of SPIONlabeled MSCs can be used to trace real-time liver healing during clinical treatment following hepatectomy. However, they did not use the MSCs to treat cirrhosis or HCC.

Bayo et al. investigated the possible relation between MSCs, autocrine motility factor (AMF) and HCC. ${ }^{15}$ The study investigated the role played by AMF in mediating the migration of MSCs, of various sources, towards human HCC. The AMF produced by HCC was found to induce the migration of different MSCs in vitro, and to enhance their metalloproteinase 2 activities. Stimulation of MSCs with recombinant AMF also induced the in vitro adhesion to endothelial cells and increased the attraction of MSCs towards HCC tumor cells. They concluded that AMF plays a role in MSCs recruitment towards HCC. Gene expression of survivin, cyclin D1 and beta-catenin were compared with and without MSCs therapy. Decrease in gene expression of cyclin D1 was observed with stem cell therapy, though it was not statistically significant when compared to both the control and the no treatment groups. However, the expression of beta-catenin was statistically significant when compared to both the control and the no treatment groups.
Beta-catenin activation has been linked to the development of HCC. ${ }^{21}$ Therefore, in this work, the decline in its expression observed with MSCs treatment might be one of the mechanisms through which MSCs exert their potential anti-tumor effect. The results of cyclin D1 and beta-catenin are in agreement with those of Abdel Aziz et al., who showed that MSCs decreased the level of gene expression of several carcinogenic genes in liver tissues after HCC induction in rats. ${ }^{13}$ They concluded that the administration of MSCs in experimental HCC models has tumor suppressive effects as evidenced by the decline in expression of Wnt signaling target genes with subsequent improvement of liver function.

The results of this work may indicate that MSCs could be labeled with nanoparticles and traced in normal liver, cirrhotic liver, and liver with HCC in animals. Labeling of cells did not affect adversely cell viability and/or proliferation. MRI is an important monitoring tool for the homing and localization of MSCs in the liver. MSCs infusion in animal models of liver cirrhosis and/or carcinoma may prove to be useful in limiting the cirrhotic and neoplastic changes. It is recommended to repeat this experiment using larger animal models, followed by its application in clinical trials for verification of its therapeutic potential.

\section{References}

1. Whittaker S, Marais R, Zhu AX. The role of signaling pathways in the development and treatment of hepatocellular carcinoma. Oncogene. 2010;29:4989-5005.

2. Seeff LB, Hoofnagle JH. Epidemiology of hepatocellular carcinoma in areas of low hepatitis B and hepatitis C endemicity. Oncogene. 2006;25:3771-3777.

3. Shah K. Mesenchymal stem cells engineered for cancer therapy. Adv Drug Deliv Rev. 2012;64:739-748

4. Qiao L, Xu Z, Zhao T, et al. Suppression of tumorigenesis by human mesenchymal stem cells in a hepatoma model. Cell Res. 2008;18:500-507.

5. Long X, Matsumoto R, Yang P, Uemura T. Effect of human mesenchymal stem cells on the growth of HepG2 and Hela cells. Cell Struct Funct. 2013;38(1):109-121.

6. Livraghi T, Meloni F, Frosi A, et al. Treatment with stem cell differentiation stage factors of intermediate-advanced hepatocellular carcinoma: An open randomized clinical trial. Oncol Res. 2005;15:399-408.

7. Ringden O, Le Blanc K. Allogeneic hematopoietic stem cell transplantation: State of the art and new perspectives. APMIS. 2005;113:813-830.

8. Lysy PA, Campard D, Smets F, Najimi M, Sokal EM. Stem cells for liver tissue repair: Current knowledge and perspectives. World J Gastroenterol. 2008;14:864-875.

9. Kim TH, Kim JK, Shim W, Kim SY, Park TJ, Jung JY. Tracking of transplanted mesenchymal stem cells labeled with fluorescent magnetic nanoparticle in liver cirrhosis rat model with 3-T MRI. Magn Reson Imaging. 2010;28:1004-1013.

10. Abdelaziz MT, Atta HM, Mahfouz $\mathrm{H}$, et al. Therapeutic potential of bone marrow-derived mesenchymal stem cellson experimental liver fibrosis. Clin Biochem. 2007;40:893-899.

11. Yanai A, Häfeli UO, Metcalfe $A L$, et al. Focused magnetic stem cell targeting to the retina using superparamagnetic iron oxide nanoparticles. Cell Transplant. 2012;21:1137-1148.

12. Babic $M$, Horak $D$, Trchova $M$, et al. Poly (L-lysine)-modified iron oxide nanoparticles for stem cell labeling. Bioconjug Chem. 2008;19:740-750.

13. Abdel Aziz MT, El Asmar MF, Atta HM, et al. Efficacy of mesenchymal stem cells in suppression of hepatocarcinorigenesis in rats: Possible role of Wnt signaling. J Exp Clin Cancer Res. 2011;30(1):49. 
14. Wang L, Deng J, Wang J, et al. Superparamagnetic iron oxide does not affect the viability and function of adipose-derived stem cells, and superparamagnetic iron oxide-enhanced magnetic resonance imaging identifies viable cells. Magn Reson Imaging. 2009;27:108-119.

15. Bayo J, Fiore E, Aquino JB, et al. Increased migration of human mesenchymal stromal cells by autocrine motility factor (AMF) resulted in enhanced recruitment towards hepatocellular carcinoma. PLOS One. 2014;9:e95171.

16. Bitar R, Leung G, Perng R, et al. MR pulse sequences: What every radiologist wants to know but is afraid to ask. Radiographics. 2006;26:513-537.

17. Papakonstantinou O, Kostaridou S, Maris T, et al. Quantification of liver iron overload by $\mathrm{T} 2$ quantitative magnetic resonance imaging in thalassemia: Impact of chronic hepatitis $\mathrm{C}$ on measurements. J Pediatr Hematol Oncol. 1999;21:142-148.

18. Yu NC, Chaudhari V, Raman SS, et al. CT and MRI improve detection of hepatocellular carcinoma, compared with ultrasound alone, in patients with cirrhosis. Clin Gastroenterol Hepatol. 2011;9:161-167.

19. Zhao S, Wang Y, Gao C, et al. Superparamagnetic iron oxide magnetic nanomaterial-labeled bone marrow mesenchymal stem cells for rat liver repair following hepatectomy. J Surg Res. 2014;191:290-301.

20. Bos C, Delmas $Y$, Desmouliere $A$, et al. In vivo MR imaging of intravascularly injected magnetically labeled mesenchymal stem cells in rat kidney and liver. Radiology. 2004;233:781-789.

21. Schmidt VA, Chiariello CS, Capilla E, Miller F, Bahou WF. Development of hepatocellular carcinoma in lqgap2-deficient mice is IQGAP1 dependent. Mol Cell Biol. 2008;28:1489-1502. 